\title{
EchoGéo
}

$54 \mid 2020$

Varia

\section{La méthode de la photo-interview à partir de la photographie aérienne}

Le cas d'un bidonville à Nanterre dans les années 1960

Laetitia Delavoipière, Sylvaine Conord et Anaïs Marshall

\section{OpenEdition}

Journals

Édition électronique

URL : https://journals.openedition.org/echogeo/20672

DOI : $10.4000 /$ echogeo.20672

ISSN : 1963-1197

Éditeur

Pôle de recherche pour l'organisation et la diffusion de l'information géographique (CNRS UMR 8586)

Référence électronique

Laetitia Delavoipière, Sylvaine Conord et Anaïs Marshall, « La méthode de la photo-interview à partir de la photographie aérienne », EchoGéo [En ligne], 54 | 2020, mis en ligne le 31 décembre 2020, consulté le 26 août 2021. URL : http://journals.openedition.org/echogeo/20672 ; DOI : https://doi.org/10.4000/ echogeo.20672

Ce document a été généré automatiquement le 26 août 2021.

EchoGéo est mis à disposition selon les termes de la licence Creative Commons Attribution - Pas d'Utilisation Commerciale - Pas de Modification 4.0 International (CC BY-NC-ND) 


\section{La méthode de la photo-interview à partir de la photographie aérienne}

Le cas d'un bidonville à Nanterre dans les années 1960

Laetitia Delavoipière, Sylvaine Conord et Anaïs Marshall

1 Cet article est issu d'une recherche élaborée dans le cadre d'un stage de première année de Master Géographie et Aménagement du Territoire' Il a été réalisé en 2019 sous la responsabilité de la sociologue Sylvaine Conord au sein du programme de recherche ALEGORIA (financé par l'ANR) initié par l'IGN (Institut national de l'information géographique et forestière) en 2017. L'objectif de ce programme est de favoriser la valorisation de fonds institutionnels iconographiques décrivant le territoire français depuis l'entre-guerres auprès du grand public. Dans ce cadre, mon travail consistait à exploiter et évaluer l'exploration visuelle des photographies aériennes dans une restitution $3 \mathrm{D}^{2}$ comme instrument de recherche et de communication en sociologie visuelle urbaine.

2 Les prémices de la photographie aérienne naissent avant les débuts de l'aviation datant de la fin du XIX siècle. En effet, Nathalie Roseau (2012) précise que ce sont «les panoramas saisis depuis les clochers et belvédères, suivis des premières prises de vue aérostatiques de Felix Nadar en 1858, puis des ascensions embarquées initiées par Henri Giffard aux Tuileries en 1878 » qui ont offert les premières vues prises du ciel. Depuis la réalisation des cartographies pour le repérage des positions ennemies pendant la Première Guerre Mondiale jusqu'à la planification urbaine de nos jours, l'utilisation de la photographie aérienne s'est notamment déployée dans les domaines de la géographie, de l'aménagement du territoire et de l'archéologie.

3 La photographie aérienne verticale présente de façon quasi exhaustive la morphologie et l'occupation du sol de l'espace photographié, mais ne représente pas la dimension sociale du territoire étudié. L'apport de cette dimension (perception et vécu) complète ainsi l'analyse territoriale, et peut être obtenue par la réalisation d'entretiens auprès de la population vivant dans les espaces photographiés. En se basant sur la méthode de John Collier, anthropologue et photographe américain, la photo-interview, qui introduisait des photographies déjà existantes ou réalisées par l'enquêteur ou l'enquêté 
lors des entretiens, notre méthode consiste à introduire des photographies aériennes lors d'entretiens pour qu'elles deviennent vecteur de discussions et d'échanges.

Notre méthode a été appliquée sur un espace ayant connu de profondes mutations urbaines et sociales au cours des cinquante dernières années : la commune de Nanterre, et plus spécifiquement deux bidonvilles particulièrement intéressants de par le peu de sources les concernant et l'intérêt pour l'équipe ALEGORIA. Il s'agit des bidonvilles « 66 et 97 rue des Prés » qui portent le nom de leur adresse. Les personnes interrogées sont d'anciens habitants de ces bidonvilles, témoins du Nanterre d'hier et d'aujourd'hui et des profondes mutations du paysage. Concernant les photographies, ce sont ainsi 174 clichés aériens, couvrant le secteur de Nanterre de 1954 à 1986, qui ont été mis à ma disposition par l'IGN et les archives départementales des Hauts-de-Seine.

Cet article propose ainsi de s'interroger sur cette méthode. Dans quelles mesures la photo-interview appliquée à la photographie aérienne complète-t-elle l'étude d'un territoire? Quels sont les apports spécifiques de la photo-interview? La réflexion s'organise en trois parties. Il s'agira tout d'abord de définir la photo-interview et d'en présenter certaines pratiques. Puis, sera explicité le déroulé de cette méthode qui a permis d'appréhender le regard d'anciens habitants de bidonville présent sur les clichés aériens qui leur a été soumis. Enfin, nous verrons ce que le récit des enquêtés a dévoilé sur le territoire étudié.

\section{La photo-interview, une méthode d'entretien autour de la photographie}

\section{Une « relation triangulaire » qui désinhibe l'enquêté}

Entretien de recherche mené sur la base d'un support photographique considéré comme susceptible de provoquer ou susciter des réactions verbales et non verbales chez la personne interviewée (Bigando, 2013), la méthode de la photo-interview est initiée par le photographe et anthropologue américain John Collier, dans les années 1960. La « relation triangulaire » (Duteil-Ogata, 2007) occasionnée par la présence de la photographie entre l'enquêteur et l'enquêté, substitué au face-à-face habituel de l'entretien, atténue «l'anxiété inhibitrice » que peut ressentir la personne interrogée qui se définit alors moins comme un «cœur de cible» (Bigando, 2013). En tant que support de discussion, les clichés présentés orientent les sujets abordés lors de l'entretien réduisant ainsi les craintes et les appréhensions, et favorisent la communication. En présentant des images des espaces de sa vie quotidienne à l'interviewé, celui-ci, initié, pourra faire ressortir des informations non visibles.

\section{Un dispositif méthodologique qui favorise la « libre expression » de l'enquêté (Bigando, 2013)}

7 Dans un ouvrage dédié à la méthodologie de la photographie comme technique de recherche, John Collier (1967) désigne les deux étapes de la photo-interview. Tout d'abord, le photo-essay consiste à sélectionner les clichés représentatifs d'une étude donnée. Ensuite, l'interview permet à l'enquêté de réagir sur chaque image. Ses réactions sont précieuses et complètent les autres matériaux de l'enquête. 
8 John Collier (1967) conseille notamment de choisir un corpus qui soit représentatif du ou des thèmes abordés. Fabienne Duteil-ogata (2007) avait par exemple choisi de présenter une vingtaine de clichés de rites religieux et de lieux de cultes shintôbouddhiques pris au Japon afin d'analyser le rôle des pratiques cultuelles dans la transmission des valeurs religieuses des immigrés japonais de Toulouse, où il n'existe pas de lieu de culte shintô. Elle proposait ainsi une narration photographique ayant pour but de mettre l'enquêté en confiance. L'auteur recommande de pratiquer l'entretien au domicile des intéressés et noter et/ou enregistrer réactions et commentaires (Duteil-Ogata, 2007).

9 Comme pour la plupart des entretiens ouverts en sciences humaines et sociales, Eva Bigando (2013) rappelle que l'enquêteur doit favoriser la prise de parole de l'enquêté en lui laissant la plus grande «liberté d'expression » possible, mais aussi la possibilité de discuter des images dans l'ordre que l'enquêté le souhaite, ordre qui pourra aussi être analysé par le chercheur.

\section{Appliquer la méthode à partir de la photographie aérienne}

\section{Interroger la mémoire vivante}

10 D'après Eva Bigando (2013), l'habitant "ordinaire ", amené à vivre quotidiennement son paysage, est le mieux placé pour évoquer sa propre sensibilité à son ordinaire paysager. Dans cette logique, j'ai cherché à appliquer cette méthode auprès de personnes résidant depuis longtemps dans le quartier étudié à Nanterre et ayant habité dans un des bidonvilles aujourd'hui disparus. Prêts à jouer le jeu de l'entretien, deux résidents m'ont été présentés par Sylvaine Conord et Alain Bocquet, trésorier de la Société d'Histoire de Nanterre.

11 A. fut le premier que je rencontrai. Je ne savais pas grand-chose de lui avant notre rendez-vous, si ce n'est qu'il avait habité au 66 rue des Prés, l'un des dix bidonvilles de Nanterre, celui où s'installaient les familles, contrairement au 97 de la même rue où vivaient les hommes seuls. Mon interlocuteur est né en Algérie en 1953. Sa famille a fui la guerre et est arrivée en France alors qu'il avait trois ans. En 1959, sa famille fait partie des premières à s'installer dans le bidonville. Elle est ensuite transférée dans la cité de transit ${ }^{3}$ Gutenberg, surnommée la « cité Blanche », construite non loin du 66, comme la plupart des familles des bidonvilles.

12 K. est né en Algérie pendant la guerre. Après avoir vécu dans la zone rurale qui sépare le Maroc et l'Algérie, l'Est marocain, puis à Oran, sa famille a suivi la vague de migrations économiques vers la France, suite à l'indépendance du pays. En 1967, alors qu'il a sept ans, sa famille s'installe au 66 rue des Prés. Arrivé à la fin du cycle du bidonville, ses parents n'ont pas construit leur propre chez eux, et ont acheté une baraque dans laquelle ils ont vécu jusqu'à la résorption du quartier en 1971 et sont évacués, comme les autres, vers une cité de transit.

13 Même si A. est de sept ans l'aîné de K., ils se connaissent. Les deux garçons ont habité le même bidonville puis la même cité de transit Gutenberg, et ont fréquenté les mêmes espaces. Les deux hommes ont une appréhension avertie du territoire et de ses 
mutations par leur connaissance du Nanterre d'hier, à travers les yeux d'enfants domiciliés dans le bidonville, et du Nanterre d'aujourd'hui en tant que Naterriens.

Ils semblent ne jamais avoir eu d'approche aérienne du secteur, mais le reconnaissent immédiatement.

\section{Des images qui renseignent sur le territoire étudié}

15 Afin de proposer, lors de l'entretien, des photographies favorisant une discussion sur le Nanterre d'hier, je me suis informée, lors des premiers contacts avec mes interlocuteurs, des lieux de leur enfance. Ensuite, je me suis renseignée sur l'histoire des bidonvilles mentionnés par les enquêtés.

D'après Muriel Cohen (2011), les premiers bidonvilles de Nanterre apparaissent entre 1952 et 1953. L'auteur en dénombre une dizaine dans les années 1960. Leur existence n'étant pas officielle, le nom des bidonvilles a des origines variées: la toponymie communale, l'origine nationale de ses occupants ou encore le nom de la rue voisine. C'est le cas du 66 rue des Prés et du 97 rue des Prés qui bordent la rue du même nom et qui s'étendent parmi les vastes espaces laissés vacants à l'époque.

En raison de l'installation des populations par interconnaissance professionnelle ou d'origine géographique, les bidonvilles ont des histoires qui diffèrent "par leur ampleur, par l'importance et l'origine de leurs habitants, par leur équipement" (Cohen, 2011, p. 4). Ceux du 66 rue des Prés et du 97 rue des Prés sont les seuls à être approvisionnés en eau et en électricité. Certains ne sont habités que par des familles, d'autres seulement par des hommes isolés, d'autres sont mixtes (Cohen, 2011).

Si certains bidonvilles sont résorbés au début des années 1960, les autres seront détruits en 1972 (Cohen, 2011). Suite à cette résorption, les habitants accèdent à des logements de qualité variable. Une petite part des familles est relogée en HLM ou accède au marché privé plus ou moins insalubre. La plus grande partie, faute de ressources économiques et relationnelles, est relogée en cités de transit à Nanterre ou Gennevilliers. Parmi elles, André Doucet qui borde le 66 puis Gutenberg, un peu plus loin, nommée par ses habitants la " cité Blanche » où s'installent la plupart des familles du 66 (entretien avec A. et K.).

19 Pour les entretiens, j'ai fait le choix d'une série de trois clichés.

20 Le premier de 1960 présente les 66 et 97 rue des Prés sur la même image et cadrés au centre (illustration 1). Cette échelle permet un plan large de l'environnement des deux bidonvilles et ainsi d'ouvrir la discussion sur les activités pratiquées autour du lieu de vie des deux hommes lorsqu'ils étaient enfants. 
Illustration 1 - Orthophoto des bidonvilles rue des Prés, 1960

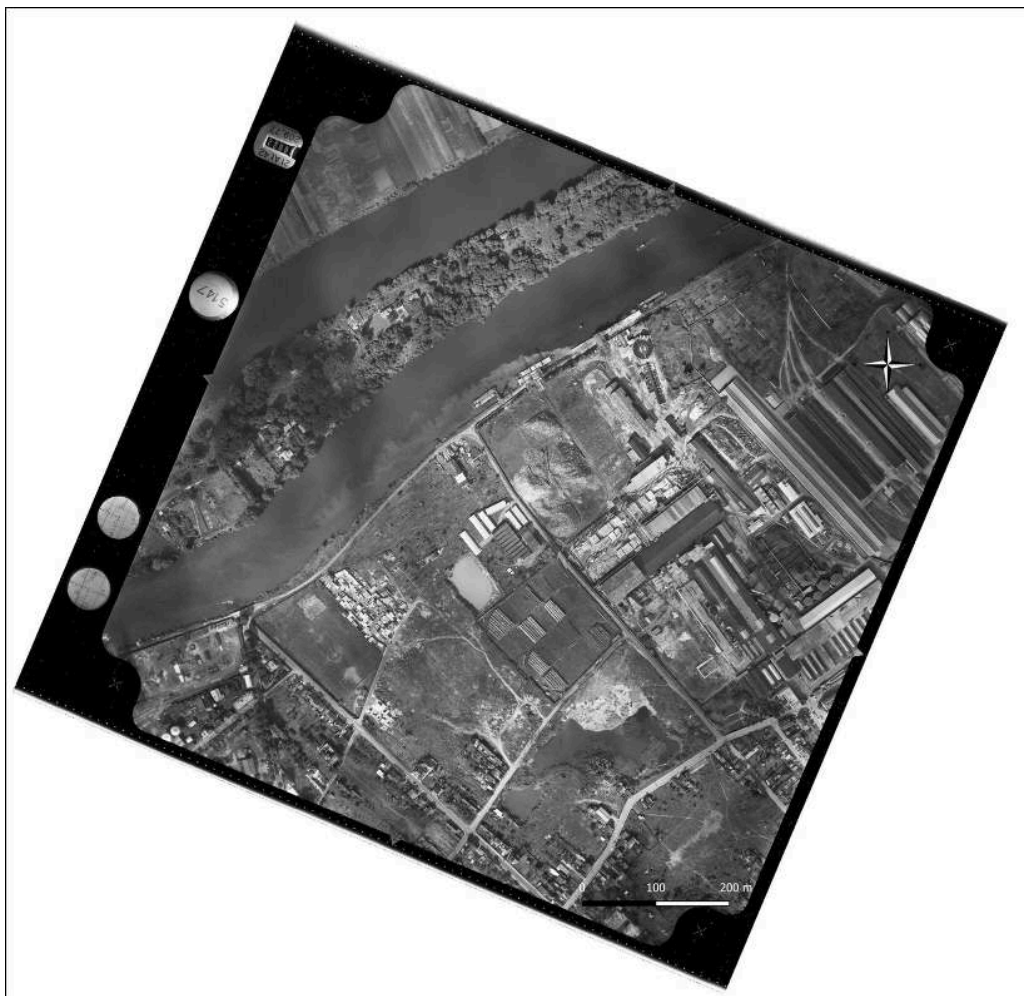

(c) IGN, 1960

21 A ce premier cliché, j'ajoute également un zoom (illustration 2) centrés sur les deux adresses laissant apparaître le proche voisinage à l'échelle du quartier. Il devrait ainsi aider à débloquer le discours sur la vie dans le bidonville. 
Illustration 2 - Orthophoto des bidonvilles rue des Prés (66 au sud, 97 au nord), zoom, 1960

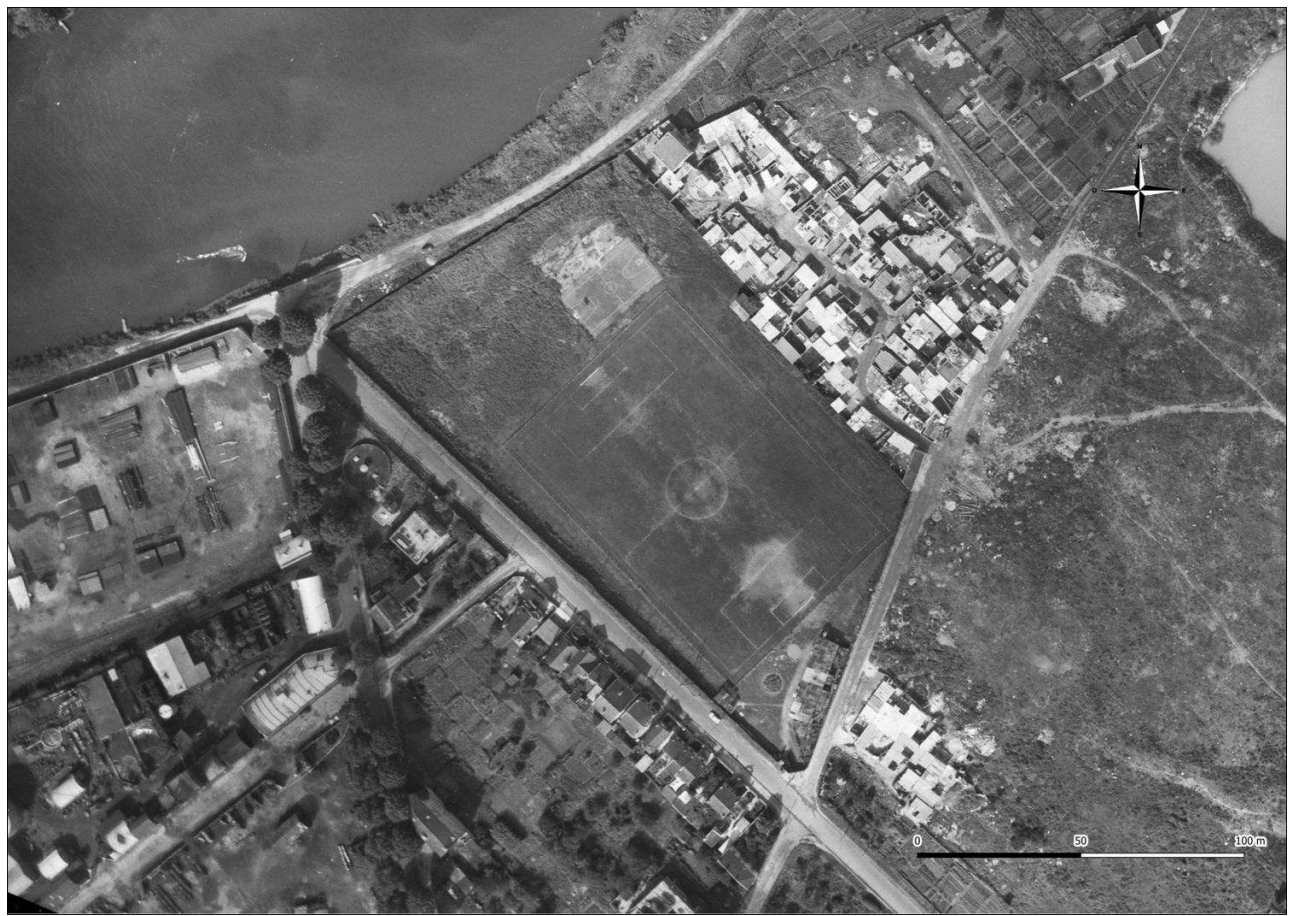

(C) IGN, 1960

Le troisième cliché date de 1971 (illsutration 3). Le bidonville n'est pas centré mais présent sous une autre forme, rasé, avec pour seuls objets deux pylônes électriques encore debout. Entre temps, la cité de transit André Doucet a vu le jour. Cette photographie permettra d'aborder l'évolution spatiale et temporelle du quartier à travers les souvenirs des interviewés. 
Illustration 3 - Orthophoto du quartier du chemin de l'île, 1971

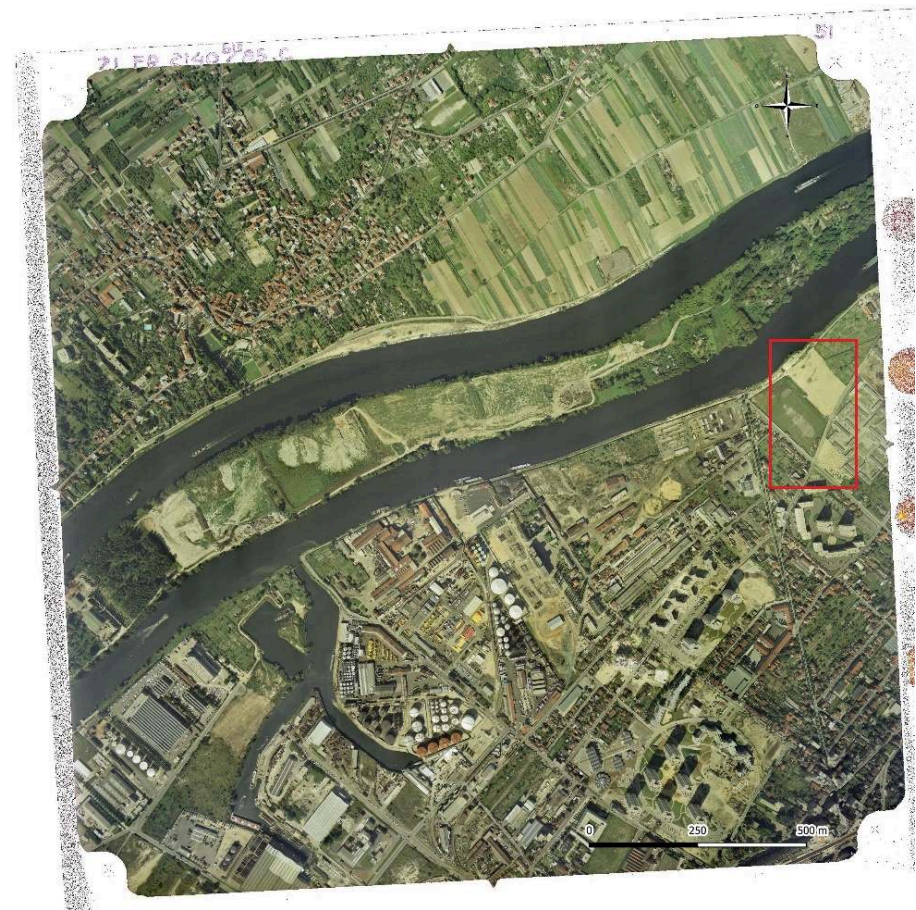

(c) IGN, 1971 (carte de localisation des clichés sur le plan actuel de Nanterre en illustration 4). Afin de laisser libre cours à d'éventuels crayonnages de leur part, une version imprimée des photos leur est fournie ainsi que des stylos-feutres de différentes couleurs. 
Illustration 4 - Situation des photographies par rapport à Nanterre

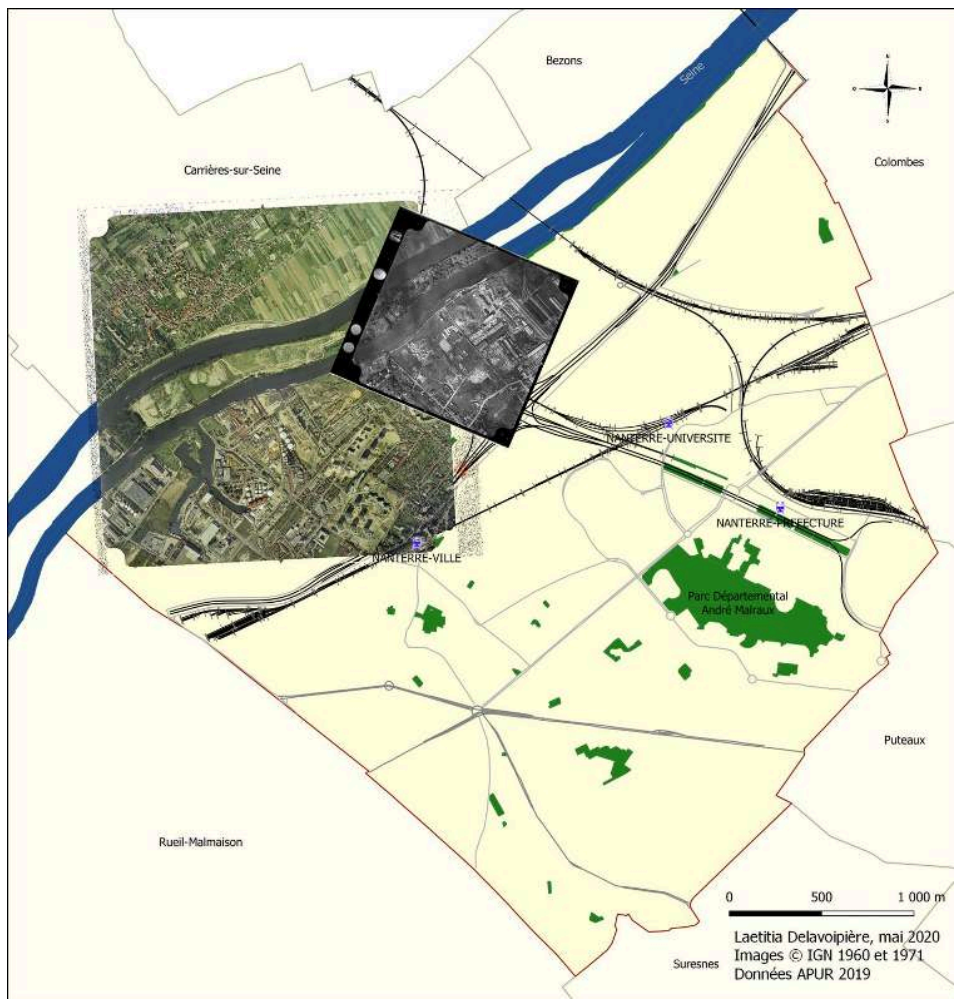

(c) IGN, 1960 et 1971. Auteur : L. Delavoipière, 2020 est-il de celui qui l'habitait? Réussira-t-il à interpréter cette vision aérienne de ces espaces de vie de son enfance ? A-t-il déjà vu une image témoignant du démantèlement de son quartier d'enfance ? Celle-ci permettra-t-elle un nouveau témoignage?

\section{L'ordre des images et le déroulement de l'entretien}

Eva Bigando (2013) fait remarquer que l'ordre proposé des photographies est chargé de sens. Celui établi dans ma recherche répondait à une logique chronologique pour que le récit soit naturel. J'ai annoncé qu'il y aurait trois images et ai attendu que la chronologie évolue pour leur présenter la dernière photographie, celle de 1971. J'avais présélectionné quelques thématiques à aborder comme le rapport à l'école ou les espaces de jeux visibles ou non sur les images. Mais les discours d'entretiens non directifs ont permis de révéler des pratiques sociales de l'espace à partir d'indices reconnaissables dans les images présentées.

J'ai convenu avec chacun des interviewés de les rencontrer dans un café de leur choix En vue de retranscrire chaque parole et chaque silence, j'ai, avec leur accord, enregistré les entretiens avec un dictaphone. 


\section{Ce que le récit dévoile sur les images}

\section{Le repérage des lieux et les aménagements invisibles}

Certains éléments de la photographie permettent la reconnaissance facile d'un lieu. Ce fut notamment le cas du stade, équipement reconnaissable en vue zénithale, situé à l'ouest du 66 rue des Prés. Cet équipement a permis à mes interlocuteurs de situer immédiatement le bidonville dans lequel ils avaient passé leur enfance. A partir de ce moment, les souvenirs ont ressurgi et par le biais d'anecdotes, un nombre important d'informations concernant l'aménagement des deux bidonvilles a été dévoilé. Les enquêtés ont annoté les photographies en indiquant le nom de voisins ou en dessinant la cité de transit André Doucet, héritière du bidonville, non construite en 1960 (date de la prise de vue) (illustration 5).

L'utilisation de la méthode de la photo-interview avec la photographie aérienne comme support a permis d'imaginer des aménagements non visibles à l'œil nu, ou encore absents au moment de la prise de vue.

Ainsi, une anecdote racontée par A., relatant ses allers et venues entre chez lui et chez un camarade habitant le 97, montre à quel point des éléments qui peuvent paraître insignifiants sont en fait riches de sens. Le manque d'éclairage public dans la rue des Prés associé aux deux seules bennes à ordures installées pour les deux bidonvilles empêchaient les habitants de voir et d'éviter les rats qui y avaient élu domicile. A. se rappelle et raconte ses craintes du danger lorsqu'il devait faire ce trajet :

« Des fois j'y allais le soir, [...] je longeais le mur [du stade] au plus près, parce que les rats, ils étaient par milliers comme ça en train de... dans les poubelles. [...] Et le problème c'est que les rats, quand ils se sentent en nombre, ils sont très méchants. Ils nous sautaient dessus. [...] Les enfants se faisaient mordre. [...] Je faisais ça dans le noir et je ne voyais rien » (entretien avec A.).

Les détails de son récit ont permis de figurer la localisation des lampadaires :

« Il y avait des poteaux d'éclairage dans cette rue-là [...], l'avenue Hoche. Donc l'éclairage [de l'avenue Hoche] arrivait jusque-là quoi. Donc c'était plus facile dans ce sens-là que dans ce sens-là » (entretien avec A.).

Illustration 5 - Cité de transit dessinée par A. à gauche, mur dessiné par K. à droite, zoom, 1960
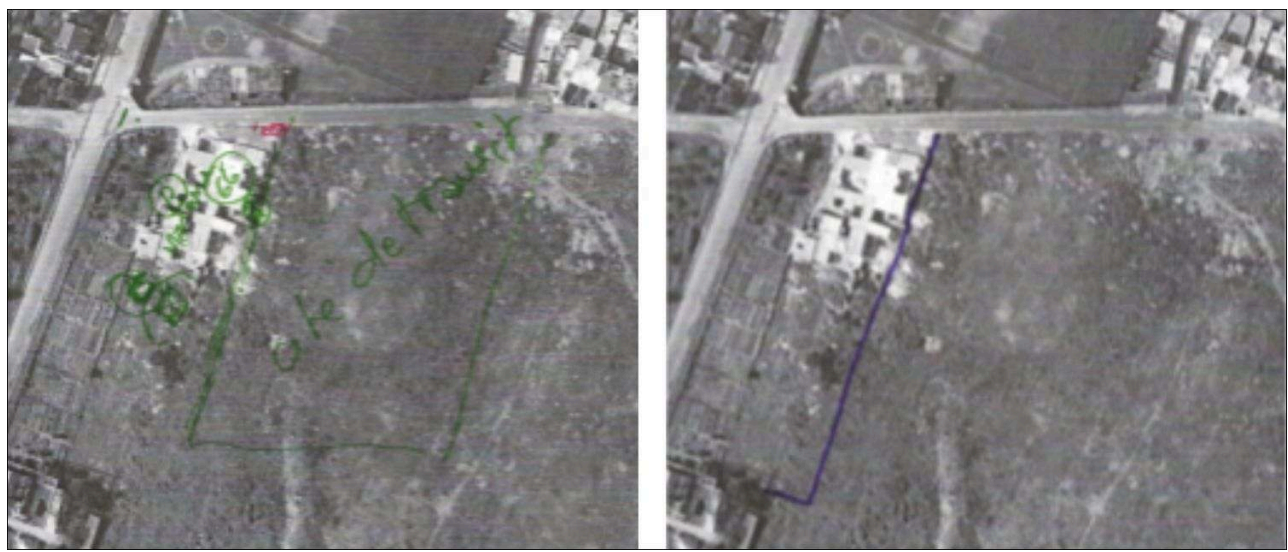

(c) IGN, 1960. 

cité de transit André Doucet pas encore construite à la date de la photographie (illustration 5).

Sans connaître la volonté des urbanistes, $\mathrm{K}$. la qualifie de « réelle séparation entre le bidonville et la cité » et ajoute des éléments nous laissant imaginer sa hauteur :

« Nous gamins, on ne voyait pas de l'autre côté. Je pense même qu'un adulte, quand il passait, il ne voyait pas de l'autre côté. Il fallait vraiment s'agripper au mur, escalader le mur pour voir ce qui se passait de l'autre côté ».

Alors qu'un mur avait été construit entre un bidonville et une cité de transit, K. relève l'absence d'un tel ouvrage entre le bidonville et la cité HLM et les pavillons situés de l'autre côté de l'avenue Hoche.

Les descriptions proposées par les deux enquêtés amènent ainsi à compléter les informations existantes concernant les aménagements du quartier me permettant de réaliser une carte à même la photographie afin de faire figurer leurs souvenirs (illustration 6).

Illustration 6 - Aménagements du quartier du Chemin de l'île entre 1952 et 1972

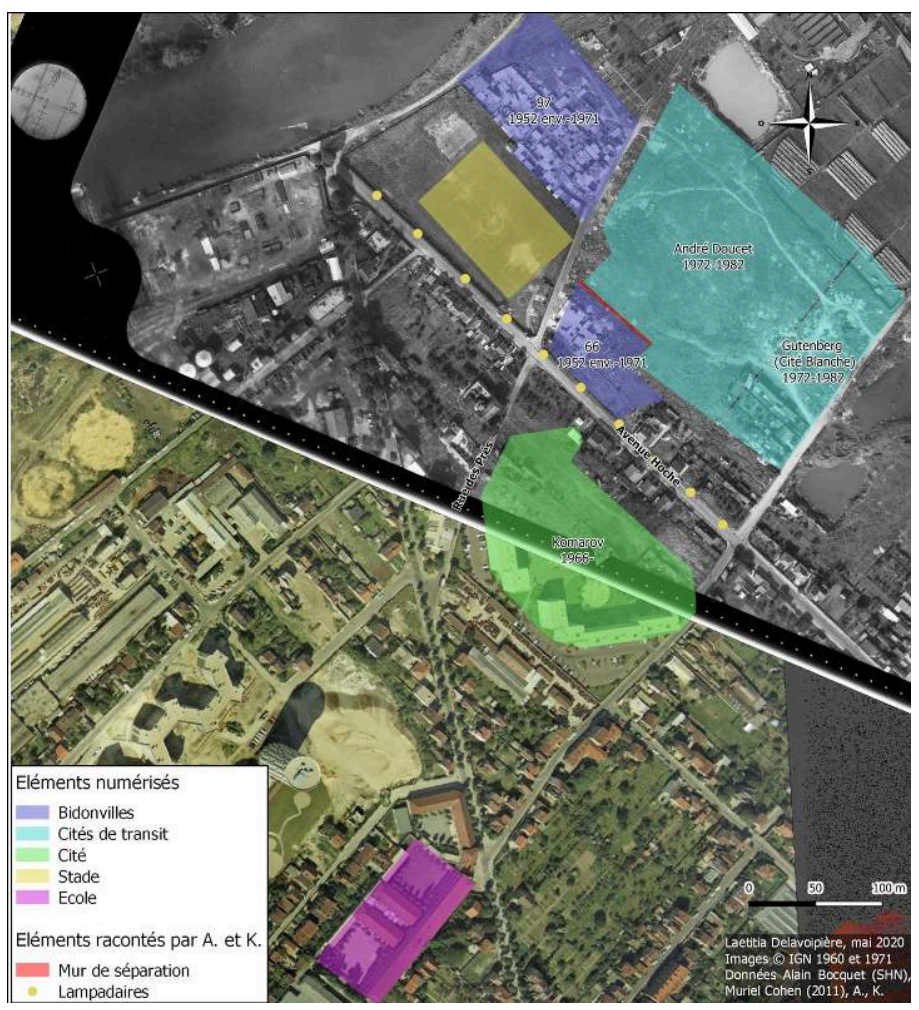

(c) IGN, 1960 et 1971. Auteur : L. Delavoipière, 2020

36 Si leurs souvenirs peuvent être biaisés par le fait qu'ils sont anciens et perçus par leurs yeux d'enfants, ils permettent de se poser des questions sur l'aménagement de l'époque. Pourquoi un mur a-t-il été construit entre une cité de transit et un bidonville et non entre ce dernier et un quartier moins précaire?

Leurs souvenirs reflètent une part de réalité vécue. Si K. se rappelle d'un mur de séparation, c'est probablement qu'il y en a vraiment eu un. Mais était-il là pour séparer les deux quartiers dans l'objectif de cacher le bidonville comme l'explique K.? «J'ose 
espérer en devenant adulte, que le mur qui était là n'était pas là pour cacher. Tu ne peux pas cacher une chose d'un côté et puis la laisser complètement de l'autre côté ». Considérant les dates de construction et de destruction des quartiers comme exactes, la cité André Doucet a vu le jour en 1972, soit approximativement un an après la destruction du 66. Le regroupement de telles informations permet de se poser de nouvelles questions et ainsi, petit à petit, trouver des pistes intéressantes. Ce mur a peut-être été bâti pour protéger les baraques des travaux de construction de la cité ?

\section{Le vécu du bidonville ou la mémoire « enchantée »} mémoire vivante. Cette dernière est ancienne et relate des événements vécus à travers leur regard d'enfants dans les années 1960. Comme le souligne Muriel Cohen (2011), le souvenir du mal-logement et de la répression meurtrière du 17 octobre 1961 n'a pas empêché ces enfants, devenus grands, d'avoir une mémoire "enchantée » de cette époque.

K. reste conscient, malgré ses souvenirs chaleureux, de l'insalubrité de son environnement d'enfance. Il se rend compte d'où proviennent ses souvenirs nostalgiques sans avoir une approche misérabiliste en se remémorant la vétusté des logements et le manque de lumière dans la rue.

Cette mémoire peut même être ponctuée d'une forme de déchirement, sentiment que semble ressentir K. lorsqu'il découvre l'image de 1971. En effet, il prend soudainement conscience, grâce à la photographie, de la disparition totale, irrémédiable et brutale d'un élément constitutif de son identité : le bidonville dans lequel il avait grandi, complètement rasé. Fabienne Duteil-Ogata (2007) évoque pareille émotion chez ses enquêtées et explique que c'est ce que Roland Barthes (1980) appelle le "punctum ", c'est-à-dire une émotion poignante causée par le hasard :

«c'est comme si on mettait du Tipp-Ex sur des centaines et des centaines de vies quoi. Y a tellement de familles qui ont vécu ici, d'enfants qui ont grouillé un peu partout ici. Et d'un seul coup, sur une photo aérienne : rien. [...] On dirait qu'on appuie sur un bouton c'est fini » (entretien avec K.).

42 A travers ses témoignages, $K$. souhaite apporter une meilleure compréhension de ce qu'étaient ces bidonvilles et la manière dont ils étaient vécus, et ainsi contribuer à faire changer les regards portés sur le bidonville qui à son sens est seulement perçu comme «sale».

Le jeune âge d'A. et $\mathrm{K}$. à l'époque leur confère aujourd'hui, de la même manière que nos propres souvenirs d'enfance, une mémoire «enchantée». Cette mémoire, bien qu'enchantée, propose néanmoins une véritable démonstration de la misère installée. Bien que perçues, ces inégalités face au logement n'étaient pas mal vécues par les deux garçons. En revanche, leur exclusion des terrains de jeux n'a pas eu le même impact.

EchoGéo, 54 | 2020 


\section{Des terrains de jeux différenciés ou les conséquences d'une ségrégation socio-spatiale} et ce malgré leur différence d'âge : il leur était interdit d'entrer dans le stade voisin de leur bidonville. A. se rappelle : «toujours le stade... Ils ne voulaient pas nous laisser rentrer. [...] Parce que nous on voulait rentrer au stade pour voir les matchs ». K. complète son témoignage en ajoutant qu'il jouait sur des terrains vagues car le "terrain de foot [leur] était interdit ", le mettant à l'écart et lui laissant le sentiment que ce mur avait été construit pour rejeter les enfants de son quartier : «On ne pouvait pas y entrer, y avait des murs, qui séparaient bien le bidonville de ce terrain de foot ». L'évocation de cette démarcation physique est confirmée par A. dans son souvenir :

«Ils nous disaient qu'il fallait payer, mais bon... on était gamin, on n'avait pas de sous. Donc qu'est-ce qu'on faisait ? [...] Y avait une barrière quoi, qui faisait peutêtre 1 mètre 80. Et tous les ballons qui arrivaient derrière, ils les revoyaient plus, hein. Donc on avait une collection de ballons. Tous les dimanches, on récupérait 2-3 ballons » (entretien avec A.).

De la même manière que K., A. raconte ses jeux d'enfants mais les replace dans leur contexte délabré, misérable. Il raconte que les enfants d'immigrés jouaient à l'école avec les enfants de la cité Komarov (qui abritait surtout des familles non-migrantes à « $99 \%$, voire à $100 \%$ ", selon $\mathrm{K}$.), mais n'avaient pas accès aux parcs de leur cité à la fin de la journée. Ils n'étaient pas protégés de la même manière, mais surtout, n'avaient pas le même terrain de jeux. Si les enfants des bidonvilles semblaient victimes de discrimination par le jeu, n'ayant pas le droit de jouer dans les parcs, ils ont pris, comme une alternative, les espaces dénués d'aménagement comme terrain de jeux. A. l'explique une fois de plus: "Ils ne nous laissaient pas rentrer jouer là [dans le parc aménagé au milieu de la cité Komarov, construite pour les «blancs ", selon lui]. Parce qu'ils avaient fait des petites aires de jeux mais ils ne nous laissaient pas rentrer. Donc bah on jouait dehors, on jouait les extérieurs ». La cité Komarov représentait pour eux une sorte de "rêve américain ", d'après les termes de $\mathrm{K}$.

Pourquoi A. et K. n'avaient-ils pas accès aux mêmes terrains de jeux que leurs camarades non issus de l'immigration ? Le parc était-il privé et était donc réservé aux habitants de la cité ? Ou est-ce que le pronom ils fait allusion aux parents de leurs camarades qui ne souhaitaient pas accueillir les enfants du bidonville?

D'après les témoignages des deux hommes, le bidonville du 66 rue des Prés répond à la définition même du ghetto expliquée par Cyprien Avenel (2009, p. 40) : «synonyme de zone de concentration de l'exclusion, dans laquelle vit une population ethniquement homogène, fonctionnant comme une microsociété, et publiquement discrédité ». De par leur quartier auto-construit et leur nationalité algérienne commune, les parents de A., de K. et de tous leurs camarades du 66 étaient probablement déjà privés de perspectives d'intégration. De la même manière que dans un imaginaire collectif d'aujourd'hui où les "jeunes de cités » sont spontanément associés à des «jeunes à problèmes " (Avenel, 2009), A. et K. ont peut-être été rejetés par les parents de leurs camarades non issus de l'immigration qui auraient considéré que les populations issues du bidonville « ont des problèmes et qu'ils sont un problème " (Avenel, 2009), les renvoyant à un statut de victimes en exerçant sur eux stigmatisation et donc exclusion. Par ailleurs, Cyprien 
Avenel rappelle que la réalité et les représentations sont certes distinctes, mais «se mêlent constamment ».

Dans leur étude sur la précarité urbaine et la fragmentation socio-spatiale dans la métropole de Recife, Jean-Paul Carrière et Luis de la Mora (2014) expliquent que les favelas ont été construites par des personnes confrontées aux mêmes problèmes de marginalisation et que cela favorise un «entre-soi» qui serait caractéristique des communautés pauvres brésiliennes. Les bidonvilles de Nanterre ont été édifiés selon le même schéma. Si parents et enfants du 66 travaillaient et étudiaient à l'extérieur du microcosme, l'« entre-soi » était probablement privilégié dans la vie quotidienne et les deux populations ne se mêlaient pas dans le cadre privé.

Les deux enquêtés se sont donc distingués des enfants qui habitaient la cité Komarov, relevant les distinctions entre les deux types d'habitat, et donnant une nouvelle fois des indications sur l'aménagement des rues:

" Et donc on allait à l'école Voltaire, et ensuite au retour à $16 \mathrm{~h} 30$ [...] on refaisait le chemin ensemble et nos copains rentraient dans la cité et nous on continuait notre chemin. [...] C'était goudronné jusqu'à l'avenue Hoche et à partir du bidonville il n'y avait plus de goudron » (entretien avec K.).

50 K. rappelle que l'avenue Hoche était goudronnée mais que la rue des Prés était boueuse par temps humide et illustre ses propos par ses habitudes quotidiennes de l'époque :

" en sortant d'ici, on avait les chaussures pleines de boue et on essayait avant d'arriver [devant] chez nos copains, nos camarades de classe qui habitaient dans des endroits un peu plus catholiques si je puis dire, on essayait d'enlever la boue de nos chaussures » (entretien avec K.).

51 L'insalubrité de la zone autour des bidonvilles, du fait de l'absence de revêtement goudronné et d'éclairage public dans la rue des Prés et le nombre restreint de bennes à ordures, propice à la multiplication des nuisibles tels que les rats, témoigne de l'installation spontanée de ces populations et de l'absence d'interventions de la part des pouvoirs publics pour régulariser et aménager les lieux. K. et $\mathrm{A}$. ont tous deux des souvenirs d'inégalités de traitement face aux enfants issus de milieux plus favorables. Ces perceptions semblent accentuées par certains aménagements comme le mur séparant le 66 de la cité André Doucet que K. qualifie de "douteux", ou par des comportements discriminatoires à l'égard des enfants des bidonvilles, comme leur souvenir d'interdiction d'entrer dans le stade ou de jouer dans les parcs de la cité Komarov.

Jean-Paul Carrière et Luis de la Mora (2014) insistent sur le fait que la fragmentation socio-spatiale de Recife n'est pas comparable avec celle des métropoles mondialisées "du nord $»^{4}$. Mais mon exploration aérienne du Nanterre de l'époque m'a fait constater une similarité. Tout comme Recife, une « mosaïque » de " morceaux de ville » formait la ville où tantôt un bidonville s'installait au milieu des champs, tantôt au pied d'immeubles et même au pied de la préfecture (illustrations 7 et 8). 
Illustration 7 - Proximité spatiale et distance sociale

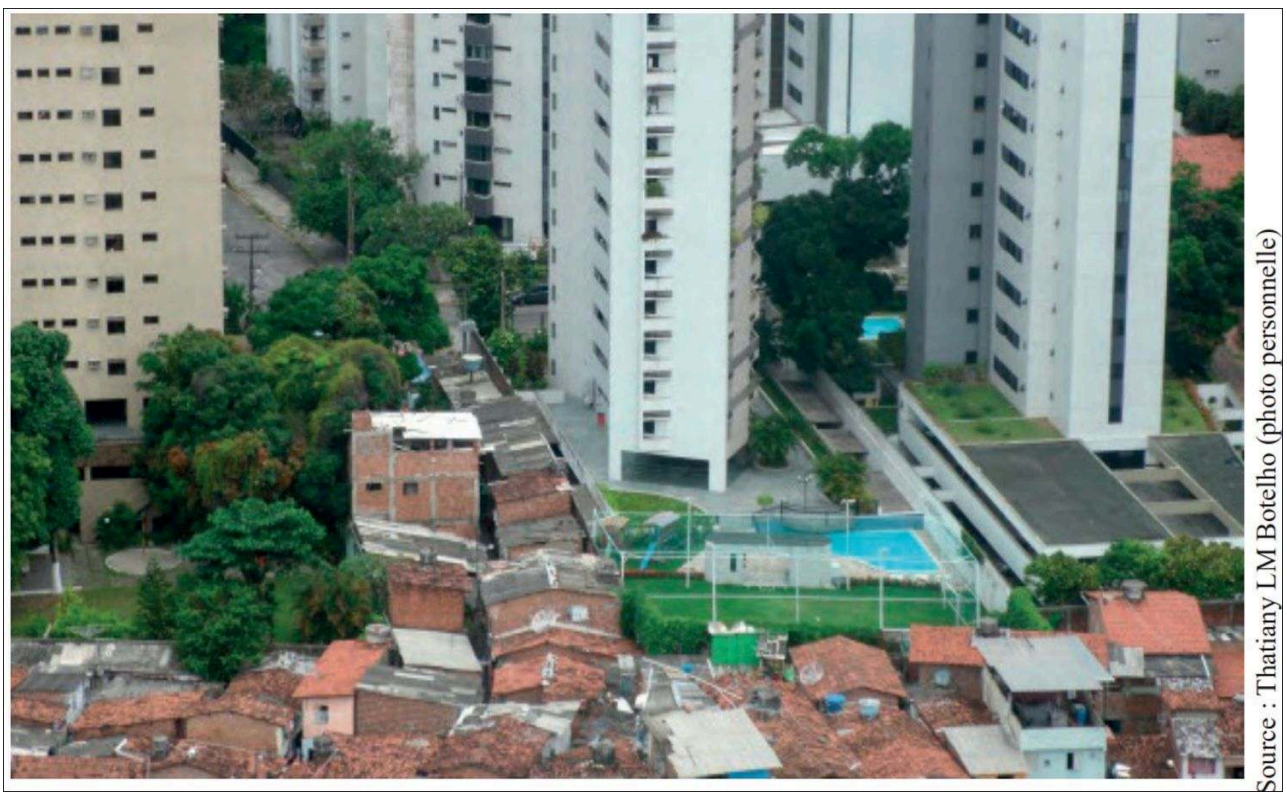

Deux mondes cloisonnés en co-présence. Des favelas au pied des condominios du quartier de Casa Forte, à Recife (Carrière et de la Mora, 2014).

(c) Thatiany LM Botelho (photo personnelle).

Illustration 8 - Bidonville des Pâquerettes, Nanterre, 1960-1961

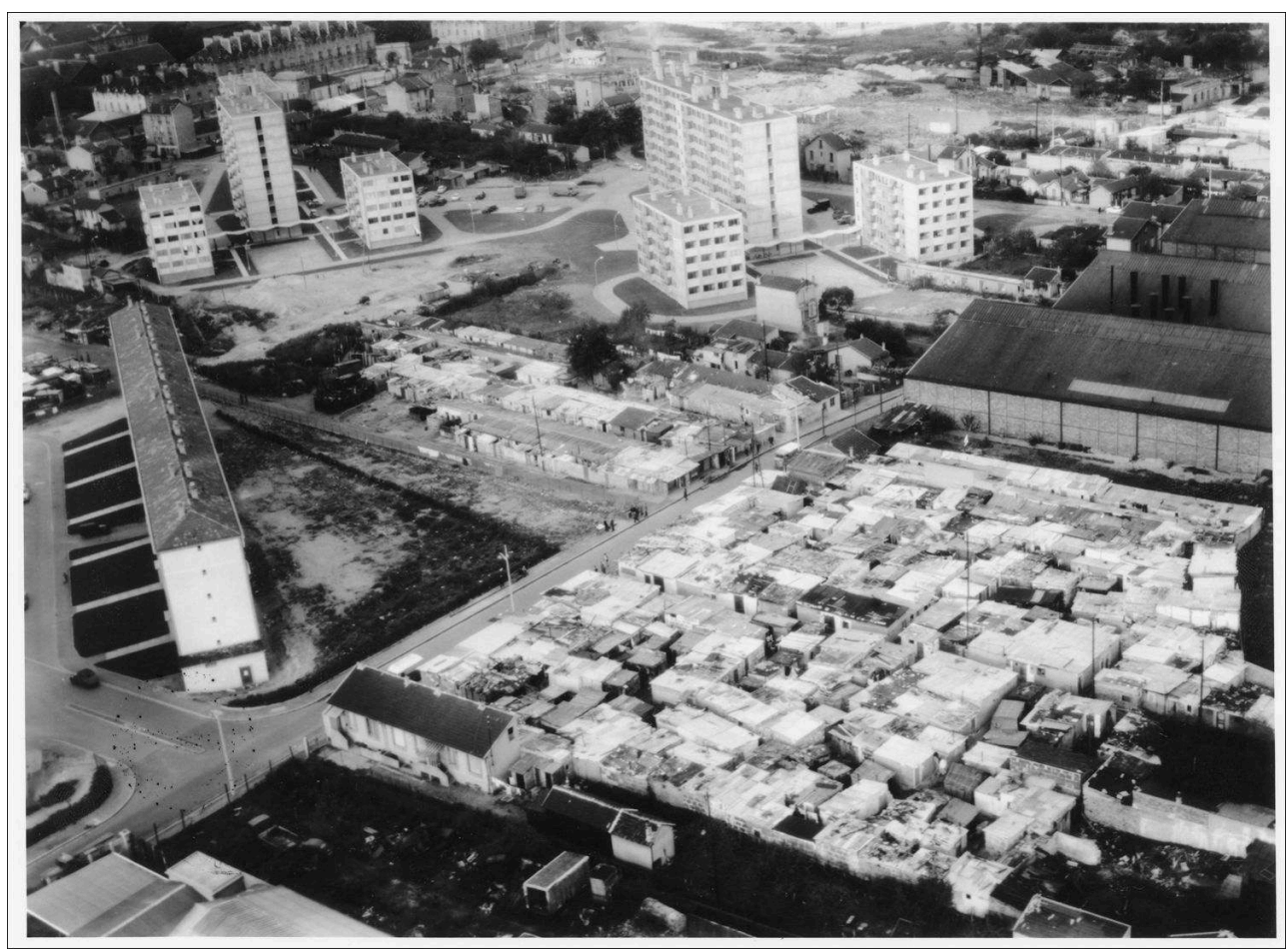

(c) $\mathrm{AD} 92$.

53 Nanterre étant encore peu urbanisée dans les années 1960, les populations immigrées, dont celles de la rue des Prés, en quête d'un espace de vie ont pu s'installer au milieu de zones non bâties. Par son accès à la rue des Prés qui donne elle-même sur l'avenue 
Hoche, le 66 forme un îlot qui n'est pas une enclave totalement fermée. La proximité entre les deux types d'habitat avec comme seule barrière concrète l'avenue Hoche crée paradoxalement une "proximité spatiale » et une "distance sociale » (Carrière, de la Mora, 2014) formant une fragmentation urbaine. Jean-Paul Carrière et Luis de la Mora (2014) ajoutent :

«Dès lors, s'impose l'image d'une ville segmentée, composant une mosaïque d'espaces identitaires, fortement différenciés par leur architecture et leurs caractéristiques paysagères, quand ils ne sont pas physiquement coupés par des barrières symboliques ou concrètes (murs, enceintes, avenues). La fragmentation laisse alors alterner, dans un voisinage sans distance spatiale, les zones d'habitat précaire et les ilots d'opulence, et paradoxalement renforce l'ancrage local des populations précarisées qui se replient sur leur espace de quotidienneté [...]» (Carrière et de la Mora, 2014).

54 L'interdiction de jouer avec leurs camarades en dehors de l'école illustre une ségrégation par le jeu due à une ségrégation socio-spatiale?

\section{Conclusion} aménagements ou les absences d'aménagement non visibles depuis le ciel. Le manque de lumière dans la rue des Prés et la crainte des rats a révélé l'absence de lampadaires et a rappelé la présence d'uniquement deux bennes à ordures pour les deux bidonvilles. Un autre souvenir d'enfance a également dévoilé la construction d'un mur entre le 66 et la cité de transit André Doucet, encore inexistant au moment de la prise de vue. photographies anciennes utilisées comme support lors de l'entretien et aide à la compréhension de l'histoire des lieux. En effet, les témoignages de ceux qui ont vécu ces bidonvilles ont apporté des informations précises sur des aménagements difficilement visibles voire non perceptibles sur un cliché aérien. Des récits qui peuvent paraître anecdotiques au départ s'avèrent être, confrontés à la photographie aérienne, un réel outil pour la création de cartes nous laissant concevoir les aménagements de l'époque et pour le témoignage d'une ségrégation socio-spatiale par le non accès à des logements formels.

Alors que Philippe Dubois (1990) définit la photographie comme une «trace », Sylvaine Conord (2007) lui attribue le rôle de mémoire d'indices qui ne sont pas tous perceptibles par le seul œil, même si elle reste subjective. Si l'image aérienne peut offrir une référence volontaire ou non, elle fait apparaître quelque chose d'autre 
qu'une simple vue du ciel. Il est difficile d'identifier un objet sociologique sur une image (Piette, 2007). En revanche, par les indices qui l'emplissent, l'image ouvre le discours de ceux qui ont vécu le paysage, permettant de «faire jaillir le remarquable [...] de rendre visuellement pertinents des traits qui ne le sont pas à partir de l'œil nu de l'acteur » et de « révéler de nombreux détails de la vie sociale » (Piette, 2007, p. 23-28). De surcroît, si les clichés peuvent aider à confirmer ou infirmer des affirmations, c'est d'autant plus le cas de la photographie aérienne.

La réalité habitante a donné vie aux formes urbaines inanimées visibles sur l'image. L'usage de la photographie aérienne ancienne comme vecteur de discussion permet à des initiés d'apporter des informations qu'un chercheur est susceptible de ne pas voir sur l'image mais peut alors imaginer d'un point de vue zénithale. Si les souvenirs sont subjectifs - et ici limités par le nombre d'enquêtés - car anciens et référant à des perceptions et ressentis propres à chacun, ils apportent des éléments inédits qui viennent renforcer la connaissance des lieux et la manière dont ils ont pu être vécus. Ces nouveaux éléments, croisés avec d'autres sources, peuvent également offrir de nouvelles pistes de réflexion.

\section{BIBLIOGRAPHIE}

Avenel C., 2009. La construction du « problème des banlieues » entre ségrégation et stigmatisation. ERES, Journal français de psychiatrie, $\mathrm{n}^{\circ} 34$, p. 36-44.

Bigando E., 2013. De l'usage de la photo elicitation interview pour appréhender les paysages du quotidien : retour sur une méthode productrice d'une réflexivité habitante. Cybergeo. European Journal of Geography [En ligne]. URL: http://journals.openedition.org/cybergeo/25919 - DOI: https://doi.org/10.4000/cybergeo.25919

Barthes Roland, La chambre claire. Notes sur la photographie, Cahiers Gallimard Seuil, 1980.

Carriere J.-P., de la Mora L., 2014. Précarité urbaine et fragmentation socio-spatiale au sein des métropoles brésiliennes : le cas de Recife ", Géographie, économie, société, vol. 16, p. 373-397.

Cohen M., 2011. Les bidonvilles de Nanterre, entre « trop-plein » de mémoire et silences. Diasporas, $\mathrm{n}^{\circ} 17$, p. 1-22.

Collier J., 1967. Visual Anthropology: Photography as a Research Method. Albuquerque, University of New Mexico Press, p. 46-66.

Conord S., 2007.Usages et fonctions de la photographie. Ethnologie française, vol. 37, p. 11-22.

Coste M., Roncayolo M., 1980. La photo-interprétation des formes urbaines, remarques d'usage. L'espace géographique, vol. 9, $\mathrm{n}^{\circ}$ 1, p. 57-69.

Delavoipière L. 2019. Comment la méthode sociologique de photo-elicitation interview associée à Itowns aide-elle l'étude géographique de la transformation du territoire de Nanterre ?. Mémoire de Master 1 Territoire et Développement Durable, Université Sorbonne Paris Nord, 73 p. Accessible en ligne : https://drive.google.com/file/d/1FybgdHbNDjF8Jhp_o2TOUF9Zx26SzL7_/view 
Dubois P., 1990. L'acte photographique. Paris, Nathan.

Duteil-Ogata F., 2007. La photo-interview : dialogues avec des Japonais. Ethnologie française, vol. 37, p. 69-79.

Piette A., 2007. Fondements épistémologiques de la photographie. Ethnologie française, vol. 37, p. 23-28.

Roseau N., 2012. La ville vue d'avion, miroir ou scénario ? Seize études pour une histoire culturelle. Vues aériennes. MetisPresses, 2012.

\section{NOTES}

1. Formation suivie au sein de l'Université Sorbonne Paris Nord.

2. Cette exploration en 3D est le résultat du prototype d'un démonstrateur développé par l'IGN : iTowns. Une partie importante de mon stage consistait à évaluer ce prototype, mais ce n'est pas l'objet de cet article. Pour plus d'informations : Delavoipière, 2019.

3. Les cités de transit sont des cités de logements provisoires construites pour quelques années dans l'attente de HLM, dont un grand nombre est resté bien plus longtemps. Comme le rappelle Muriel Cohen, l'idée qui prévalait à l'époque était que les Algériens étaient incapables d'habiter un logement moderne et qu'il fallait leur « apprendre » à " se civiliser » en résidant deux années en cité de transit. Elle accuse une « hypocrisie totale » car les familles concentrées en fonction de leurs origines ethniques dans des ghettos étaient déjà exclues de la population française. Elle ajoute que certaines familles sont restées 18 ans en cité de transit, et que les dernières ont disparu en 1985. (Constantinesco Laure - TV5 Monde, 2011. Muriel Cohen, historienne, raconte les bidonvilles en France. Entretien retranscrit sur le site de TV5 Monde le 9/09/2001 et mis à jour le 08/05/2012. https://information.tv5monde.com/info/muriel-cohen-historienne-raconte-lesbidonvilles-en-france-3460)

4. «Les grandes villes brésiliennes, à l'instar de ce que l'on peut voir dans beaucoup de pays du sud (Bretagnolle et al., 2011), connaissent une exacerbation de leurs contrastes en termes de niveau de vie, de qualité de bâti, d'accès à l'emploi et aux services... Le renforcement des inégalités à l'échelle infra-métropolitaine dans ce pays est à l'origine de processus de fragmentation socio-spatiale, sans commune mesure avec ce que l'on peut connaître dans les métropoles mondialisées du "nord », européennes ou nord-américaines, la manifestation la plus tangible en étant la dissémination et l'extension continue des favelas au cœur même des villes » (Jean-Paul Carrière et Luis de la Mora, 2014).

\section{RÉSUMÉS}

La photographie aérienne présente de façon quasi exhaustive la morphologie et l'occupation du sol de l'espace photographié, mais ne représente pas la dimension sociale du territoire étudié. Cet article évoque un exemple d'usage particulier de la méthode de la photo-interview consistant à introduire des photographies lors d'entretiens pour qu'elles deviennent vecteur de discussions et d'échanges. Adaptée cette fois-ci à la photographie aérienne, cette technique d'enquête a été expérimentée auprès de deux anciens habitants d'un bidonville de Nanterre. Le recueil des 
réactions aux images des espaces qu'ils avaient vécu a permis d'enrichir l'analyse territoriale dans un travail de recherche sur l'urbanisation précaire de Nanterre.

Aerial photography presents almost exhaustively the morphology and land use of the photographed space, but does not represent the social dimension of the studied territory. This article mentions an example of a particular use of the photo-interview method introducing pictures during interviews so that they become a vector for discussions and exchanges. Adapted to aerial photography, this investigative technique was tested with two former residents of a slum in Nanterre. The collection of reactions to the images of the spaces they had lived has enriched the territorial analysis in a research work on the precarious urbanization of Nanterre.

\section{INDEX}

Keywords : photo-interview, aerial photography, slums, Nanterre, 1960's, "enchanted" memory, socio-spatial segregation

Mots-clés : photo-interview, photographie aérienne, bidonvilles, Nanterre, années 1960, mémoire " enchantée ", ségrégation socio-spatiale

\section{AUTEURS}

\section{LAETITIA DELAVOIPIÈRE}

Laetitia Delavoipière, laetitia.delavoipiere@laposte.net, est étudiante en master 2 à l'Université Sorbonne Paris Nord, Villetaneuse. Elle a récemment publié :

- Lecat P., Blettery E., Delavoipière L., Gouet-Brunet V., Conord S., Saly-Giocanti F., Devaux A., Bredif M., Moret F., 2020. Restituer les bidonvilles de Nanterre. Retour d'expérience d'un outil de visualisation 3D des données spatiales à l'usage des sciences sociales. Humanités numériques [En ligne], à paraître. URL: https://journals.openedition.org/revuehn/

- Blettery E., Lecat P., Devaux A., Gouet-Brunet V., Saly-Giocanti F., Bredif M., Delavoipière L., Conord S., Moret F., 2020. A spatio-temporal web application for the understanding of the formation of the parisian metropolis. SPRS Annals of the Photogrammetry, Remote Sensing and Spatial Information Sciences, vol. $4, \mathrm{n}^{\circ} 4$.

\section{SYLVAINE CONORD}

Sylvaine Conord, sylvaineconord@orange.fr, est maîtresse de conférences à l'Université Paris Nanterre et membre de l'UMR LAVUE (CNRS, 7218). Elle a récemment publié :

- Lecat P., Blettery E., Delavoipière L., Gouet-Brunet V., Conord S., Saly-Giocanti F., Devaux A., Bredif M., Moret F., 2020. Restituer les bidonvilles de Nanterre. Retour d'expérience d'un outil de visualisation 3D des données spatiales à l'usage des sciences sociales. Humanités numériques [En ligne], à paraître. URL: https://journals.openedition.org/revuehn/

- Blettery E., Lecat P., Devaux A., Gouet-Brunet V., Saly-Giocanti F., Bredif M., Delavoipière L., Conord S., Moret F., 2020. A spatio-temporal web application for the understanding of the formation of the parisian metropolis. SPRS Annals of the Photogrammetry, Remote Sensing and Spatial Information Sciences, vol. $4, \mathrm{n}^{\circ} 4$.

- Conord S., 2017. Pour une approche collaborative en sociologie visuelle. Lorsque les enquêtés deviennent guides du chercheur-photographe. Revue françaises des méthodes visuelles [En ligne], $\mathrm{n}^{\circ}$ 1. URL: https://rfmv.fr/numeros/1/articles/une-approche-collaborative-en-sociologievisuelle-lorsque-les-enquetes-deviennent-guides-du-chercheur-photographe/ 


\section{ANAÏS MARSHALL}

Anaïs Marshall, anais.marshall@univ-tlse2.fr, est maîtresse de conférences à l'Université Toulouse - Jean Jaurès et membre de l'UMR LISST-Dynamiques rurales et associée à l'EA Pléiade. Elle a récemment publié :

- Lavie E., Crombé L., Marshall A., 2020. Reconceptualising the drinking waterscape through a grounded perspective. Geographical Journal [En ligne], vol. 186, n² 2. DOI: https://doi.org/10.1111/ geoj.12343

- Lavie E., Marshall A., 2019. Avant-postes et résistances à l'universalisation d'un modèle urbain de service public en zone rurale : le cas de l'accès à l'eau potable en périphérie de Mendoza (Argentine). Annales de géographie, $\mathrm{n}^{\circ}$ 725, p. 5-30.

- Marshall A., Alexandre F., 2018. Des lieux qui «n'existent » pas : regard de géographes sur le projet Llocs que no existeixen (Goggle earth 1.0) (Isaki Lacuesta et Isa Campo, 2009). In Castann Akrami B., Ramos Alquzar S., Le cinéma d'Isaki Lacuesta. Coéditions Mare et Martin Arts/Centre Pompidou, $262 \mathrm{p}$. 